\title{
FIXED POINTS OF AUTOMORPHISMS OF COMPACT RIEMANN SURFACES AND HIGHER-ORDER WEIERSTRASS POINTS
}

\author{
RYUTARO HORIUCHI AND TOMIHIKO TANIMOTO
}

(Communicated by Irwin Kra)

Dedicated to Professor Kôtaro Oikawa for his 60th birthday

\begin{abstract}
A sufficient condition for fixed points of an automorphism of prime order on a compact Riemann surface to be higher-order Weierstrass points is given. This leads us to a complete study of the cases where the prime orders are small.
\end{abstract}

1. Let $M$ be a compact Riemann surface of genus $g \geq 2$. We denote Aut $M$ the group of conformal automorphisms of $M, \nu(T)$ the number of fixed points of an automorphism $T \in$ Aut $M$ and $H^{q}(M)$ the space of holomorphic $q$-differentials on $M$.

Lewittes proved that if $\nu(T) \geq 5$, then every fixed point is a 1 -Weierstrass point [5], and in this relation, some cases have been studied by Accola [1], Duma [2], Farkas and $\mathrm{Kra}$ [3] for higher-order Weierstrass points (see Corollaries 1, 2, 3,4 below). Guerrero [4] proved that if $\nu(T)=1$ and the fixed point is not a 1-Weierstrass point, then $T$ has order $6, g \equiv 1 \bmod 6$ and the fixed point is a $q$-Weierstrass point for all $q \geq 2$. It is known that if the order of $T$ is prime, then $\nu(T) \geq 2$ [3]. Guerrero also gave examples of Riemann surfaces with automorphisms of prime order whose two fixed points are not $q$-Weierstrass points for $q \geq 2$.

The purpose of this paper is to give a sufficient condition for fixed points to be $q$-Weierstrass points $(q \geq 2)$ and to supplement the results mentioned above. We will show that if $\nu(T)(2 s+1-n) \neq 2(n \delta-r)$, then the fixed points of $T$ are $q$-Weierstrass points, and study the case where $\nu(T) \geq 3$ and the order of $T$ is 5 .

2. For $T \in$ Aut $M$, let $\varepsilon$ be the rotation constant of $T$ at a fixed point of $T$, i.e. locally $T^{-1}: z \rightarrow \varepsilon z$. There is a basis for the space of holomorphic $q$-differentials such that the linear map induced by $T$ on this space is given by the matrix $\operatorname{diag}\left(\varepsilon^{\gamma_{1}-1+q}, \varepsilon^{\gamma_{2}-2+q}, \ldots, \varepsilon^{\gamma_{d}-1+q}\right)$ for each $q \geq 2$, where $d=$ $(2 q-1)(g-1)$, and $1=\gamma_{1}<\gamma_{2}<\cdots<\gamma_{d}<2 q(g-1)+2$ is the q-gap

Received by the editors June 27, 1988 and, in revised form, August 29, 1988.

1980 Mathematics Subject Classification (1985 Revision). Primary 14F07; Secondary 30F 10. 
sequence at the fixed point. If there exists such a $\gamma_{j}$ with $\gamma_{j}>j$ for at least one $j$ in the $q$-gap sequence at a point, then the point is called a $q$-Weierstrass point. So, for a fixed point which is not a $q$-Weierstrass point, we have the matrix

$$
\operatorname{diag}\left(\varepsilon^{q}, \varepsilon^{q+1}, \ldots, \varepsilon^{d-1+q}\right) .
$$

The multiplicity of the eigenvalue 1 is equal to $\operatorname{dim} H_{\langle T\rangle}^{q}(M)$ (for more details, see Farkas and Kra [3]).

3. Now we give a sufficient condition for fixed points to be $q$-Weierstrass point $(q \geq 2)$.

Theorem 1. Assume that for $T \in$ Aut $M$ of prime order $n$, there is a fixed point of $T$ which is not a $q$-Weierstrass point for some $q \geq 2$. Let $q-1=k n+s$ $(0 \leq s \leq n-1), g-1=m n+t \quad(0 \leq t \leq n-1),(2 q-1)(g-1)=$ $[(2 q-1)(g-1) / n] n+r(0 \leq r \leq n-1)$ and $\delta=[(r+s) / n]$. Then we have

$$
\nu(T)(2 s-(n-1))=2(n \delta-r) .
$$

Proof. The representation of $T$ on $H^{q}(M)$ is

$$
\operatorname{diag}\left(\varepsilon^{q}, \ldots, \varepsilon^{(2 q-1)(g-1)+q-1}\right),
$$

where $\varepsilon=e^{2 \pi i / n}$, and the multiplicity of the eigenvalue 1 is

$$
\left[\frac{(2 q-1)(g-1)+q-1}{n}\right]-\left[\frac{q-1}{n}\right],
$$

and is also equal to $\operatorname{dim} H_{\langle T\rangle}^{q}(M)$.

We set

$$
\begin{aligned}
\delta^{\prime} & =\left(\left[\frac{(2 q-1)(g-1)+q-1}{n}\right]-\left[\frac{q-1}{n}\right]\right)-\left[\frac{(2 q-1)(g-1)}{n}\right] \\
& =\left[\frac{(2 s+1) t+s}{n}\right]-\left[\frac{(2 s+1) t}{n}\right] .
\end{aligned}
$$

Now we have

$$
(2 q-1)(g-1)=\left[\frac{(2 q-1)(g-1)}{n}\right] n+r \quad(0 \leq r \leq n-1),
$$

and

$$
(2 q-1)(g-1) \equiv(2 s+1) t \equiv r \quad(\bmod n) .
$$

If we write $(2 s+1) t=p n+r$, then

$$
\delta^{\prime}=\left[\frac{p n+r+s}{n}\right]-\left[\frac{p n+r}{n}\right]=\left[\frac{r+s}{n}\right],
$$

and thus we have $\delta=\delta^{\prime}=0$ or 1 .

Substituting the relation

$$
\begin{aligned}
{\left[\frac{(2 q-1)(g-1)}{n}\right] } & =\operatorname{dim} H_{\langle T\rangle}^{q}(M)-\delta \\
& =(2 q-1)(\tilde{g}-1)+\nu(T)[q(1-1 / n)]-\delta,
\end{aligned}
$$


where $\tilde{g}$ is the genus of the Riemann surface $M /\langle T\rangle$ and the Riemann-Hurwitz formula

$$
g-1=n(g-1)+\frac{1}{2} \nu(T)(n-1)
$$

into the relation (2), we get

$$
\nu(T)(2 s-(n-1))=2(n \delta-r) .
$$

4. In the case $n=2$, under the same hypothesis as in the above theorem, we have $\nu=2$, which means that the genus $g \equiv 0 \bmod n$ as is seen from the Riemann-Hurwitz relation. In the case $\nu=2$, it was shown by Guerrero [4] that there exists an automorphism of prime order $n$ on a Riemann surface of genus $n$ whose two fixed points are not $q$-Weierstrass points $(q \geq 2)$.

Corollary 1 (Duma [2]). Let $T \in$ Aut $M$ be of order 2. If $\nu(T) \geq 3$, then every fixed point is a $q$-Weierstrass point $(q \geq 2)$.

Corollary 2 (Farkas and Kra [3]). Let $T \in$ Aut $M$ be of prime order $n$. If $\nu(T) \geq 3$, then every fixed point of $T$ is a $q$-Weierstrass point for $q \geq 2, q \equiv 1$ $(\bmod n)$.

Proof. If we set $s=0$ in Theorem 1 , then $\delta=[(r+s) / n]=0$ so that $\nu(T)=$ $2 r /(n-1) \leq 2$. This contradiction proves that the fixed points are $q$-Weierstrass points with $q \equiv 1 \bmod n$.

Corollary 3 (Accola [1]). Let $T \in$ Aut $M$ be of prime order $n$. If $\nu(T) \geq 3$, then every fixed point of $T$ is an $n$-Weierstrass point.

Proof. If we set $s=n-1$ in the above theorem, then

$$
\nu(T)=2(n \delta-r) /(n-1) \leq 2 .
$$

This cotradiction shows that fixed points are $q$-Weierstrass points with $q \equiv$ $0 \bmod n$.

5. Now we can improve Corollary 2 and Corollary 3 to some extent:

Theorem 2. Let $T \in$ Aut $M$ be of prime order $n \geq 3$. If $\nu(T) \geq 3$, then every fixed point of $T$ is a $q$-Weierstrass point for $q \geq 2, q-1 \equiv s(\bmod n)$, where $s$ satisfies the inequalities

$$
\frac{\nu}{2(\nu-1)}(n-1)<s \quad \text { or } \quad s<\frac{\nu-2}{2(\nu-1)}(n-1) .
$$

Proof. If $\delta=0$ in (1), then we have $\nu(T)(n-1-2 s)=2 r \geq 0$ and $\nu(T)(n-1)-2(\nu(T)-1) s=2(r+s) \leq 2(n-1)$ so that

$$
\frac{\nu(T)-2}{2(\nu(T)-1)}(n-1) \leq s \leq \frac{n-1}{2} \text {. }
$$

If $\delta=1$ in (1), then we have

$$
\nu(T)(2 s-(n-1))=2(n-r)>0
$$


and

$$
2(\nu(T)-1) s-\nu(T)(n-1)=2(n-(r+s)) \leq 0
$$

so that

$$
\frac{n}{2} \leq s \leq \frac{\nu(T)}{2(\nu(T)-1)}(n-1) .
$$

Since $(\nu(T)-2) / 2(\nu(T)-1) \leq 1 / 2$ and $\frac{1}{2} \leq \nu(T) / 2(\nu(T)-1)$, the theorem is now proven.

From this theorem, we can obtain the following:

Corollary 4 (Duma [2]). Let $T \in$ Aut $M$ be of order 3. If $\nu(T) \geq 3$, then every fixed point is a $q$-Weierstrass point $(q \geq 2)$ except for $q \equiv 2(\bmod 3)$.

The remaining case $q \equiv 2 \bmod 3$ will be settled, following Guerrero's example [4].

The hyperelliptic Riemann surface defined by the equation

$$
w^{2}=\left(1+z^{3}+z^{6}+z^{9}\right),
$$

has genus $g=4$, and has an automorphism with three fixed points, two of which over $z=0$ can be shown to be non-5-Weierstrass points.

6. As for the case $n=5$, the cases $s=0$ and $s=4$ are settled by Theorem 2 .

In the case $s=2$, there exists a hyperelliptic Riemann surface of genus 5 with an automorphism of order 5 whose two fixed points are not $q$-Weierstrass points $(q \equiv 3 \bmod 5)$ (Guerrero [4]).

In the case $s=1$, assume that a fixed point of $T$ is not a $q$-Weierstrass point, then we have $\nu(T)=r=3$, provided that $\nu(T) \geq 3$.

In the case $s=3$, we have $\nu(T)=3, r=2$ under the same assumptions as in the case $s=1$.

We can show that the hyperelliptic Riemann surface defined by the equation

$$
w^{2}=1+z^{5}
$$

is of genus two and has three fixed points, two of which over $z=0$ are not $q$-Weierstrass points for $q=4,7$.

Thus we have the next corollary.

Corollary 5. Let $T \in$ Aut $M$ be of order 5. If $\nu(T) \geq 3$, then every fixed point is a $q$-Weierstrass point $(q \geq 2)$, except for the following cases:

(1) $q \equiv 2 \bmod 5$ and $\nu(T)=r=3$,

(2) $q \equiv 3 \bmod 5$

(3) $q \equiv 4 \bmod 5$ and $\nu(T)=3, r=2$.

\section{REFERENCES}

1. R. D. M. Accola, On generalized Weierstrass points on Riemann surfaces, Modular functions in analysis and number theory, 1-19, Lecture Notes Mathematics Statistics, 5, University of Pittsburgh, Pittsburgh, PA, 1983.

2. A. Duma, Holomorphe Differentiale höherer Ordnung auf kompakten Riemannschen Flächen, Schriftenreihe der Univ. Münster, 2, Serie, Heft 14, 1978. 
3. H. M. Farkas and I. Kra, Riemann surfaces, Graduate texts in Math. 71, Springer-Verlag, New York, 1980.

4. I. Guerrero, Automorphisms of compact Riemann surfaces and Weierstrass points, Riemann Surfaces and Related Topics, Proceedings of the 1978 Stony Brook Conference, Princeton University Press, Princeton, NJ, 1980.

5. J. Lewittes, Automorphisms of compact Riemann surfaces, Amer. J. Math. 85 (1963), 732-752.

Department of Mathematics, Kyoto Sangyo University, Kamigamo Kitaku Kyoto 603, JAPAN 